\title{
80. A Population Genetic Model of the Evolution of Oblique Cultural Transmission
}

\author{
By Kenichi AokI \\ National Institute of Genetics, Mishima, Shizuoka-ken 411 \\ (Communicated by Motoo Kimura, M. J. A., Oct. 12, 1984)
}

In man, and to a lesser degree in some other vertebrates, ${ }^{1) .21}$ behavior is determined by culture as well as genes. The essence of culture as defined by evolutionary biologists is information transmitted non-genetically, more specifically by observational learning. ${ }^{1 \text { (-i) }}$ Culturally determined behavior shows considerable plasticity, but the capacity for culture itself must be a genetically determined trait that evolved because of the advantage it conferred and that is constrained by that fact. In this note, I present a simple population genetic model of the evolution of cultural transmission. This model was motivated by the recent theoretical work of Cavalli-Sforza and Feldman $^{(6)}$ who investigated conditions under which cultural determination of phenotype is expected to be more adaptive than innate determination. As an application, I show that, perhaps paradoxically, contrariness may sometimes be superior to imitativeness. A fuller presentation including an application to Cavalli-Sforza and Feldman's original problem will be given elsewhere.

Let there be two haploid asexual genotypes $A$ and $a$, each capable of expressing either of two phenotypes 1 and 2 . The fitness of an individual is determined by its phenotype, but the probability of expressing a particular phenotype is dependent on genotype (see below). We assume viability selection with selection coefficient $s(>0)$ against phenotype 2. For example, phenotype 1 may be skilled and phenotype 2 unskilled. Among adults after viability selection, denote the frequency of inaividuals of genotype $A$ by $p$ and phenotype 1 by $y$. I assume that the probability of acquiring a cultural phenotype depends on the frequency of that phenotype in the adult population of the previous generation (oblique transmission ${ }^{4)(6)}$ ). In particular, I assume no direct parental influence on phenotype (vertical transmission $^{(+.6)}$ ). Thus, the frequencies of the four phenotype genotype ccmbinations $^{7}$ ) need not be considered explicitly. A newborn of the next generation is of the same genotype as its single parent. Its phenotype is determined as follows: If of genotype $A$, it expresses phenotype 1 with probability $f(y)$ and phenotype 2 with probability $1-f(y)$; if of genotype $a$, it expresses phenotype 1 with probability 
$g(y)$ and phenotype 2 with probability $1-g(y)$. Then, with discrete non-overlapping generations, our basic coevolutionary equations are

$$
\begin{gathered}
\bar{W} y^{\prime}=p f(y)+(1-p) g(y) \\
\bar{W} p^{\prime}=p f(y)+(1-s) p[1-f(y)],
\end{gathered}
$$

where a prime indicates the next generation and $\bar{W}$ is the mean fitness given by

$$
\bar{W}=1-s[1-p f(y)-(1-p) g(y)] .
$$

Putting $\Delta y=y^{\prime}-y$ and $\Delta p=p^{\prime}-p$, Eqs. (1a) and (1b) can be written alternatively as

$$
\begin{gathered}
\bar{W} \Delta y=-y(1-s)+(1-s y)[p f(y)+(1-p) g(y)] \\
\bar{W} \Delta p=s p(1-p)[f(y)-g(y)] .
\end{gathered}
$$

The equilibria can be obtained by setting the right hand sides of Eas. (3a) and (3b) simultaneously equal to 0 . Local stability of an equilibrium can be tested by obtaining the maximal eigenvalue of the following matrix:

$$
\left(\begin{array}{cc}
{\left[\hat{p} f^{\prime}(\hat{y})+(1-\hat{p}) g^{\prime}(\hat{y})\right](1-s \hat{y})} & {[f(\hat{y})-g(\hat{y})](1-s \hat{y})} \\
\hat{\hat{W}} & \hat{\bar{W}} \\
s \hat{p}(1-\hat{p})\left[f^{\prime}(\hat{y})-g^{\prime}(\hat{y})\right] & \frac{1-s[1-(1-\hat{p}) f(\hat{y})-\hat{p} g(\hat{y})]}{\hat{W}} \\
\hat{\bar{W}} & \frac{\hat{W}}{}
\end{array}\right)
$$

where a prime here indicates differentiation by $y$ and a caret indicates the equilibrium value. A convenient condition for global stability considerations can be obtained from (1a) and (2). This is

$$
\bar{W}(y, p)\left(1-s y^{\prime}\right)=1-s,
$$

where we have made the dependence of $\bar{W}$ on $y$ and $p$ explicit. Thus, using a prime and a double prime to indicate frequencies in the next generation and two generations hence, respectively, we have that $\bar{W}\left(y^{\prime}, p^{\prime}\right)-\bar{W}(y, p)$ and $y^{\prime \prime}-y^{\prime}$ are of the same sign. Unfortunately, this condition cannot be applied to the particular problem considered below.

The problem is whether a mild form of non-conformism which I call contrariness can be more adaptive than the simplest form of closervational learning, i.e. imitativeness. Imitativeness means that a culturally naive individual copies a randomly observed individual of the parental generation, whereas contrariness involves adoption of the alternative phenotype. Let genotype $A$ be imitative and genotype $a$ be contrary. We make the reasonable assumption that acquisition of the skilled phenotype, i.e. phenotype 1 , is error prone ${ }^{6)}$ with error rates $u$ and $v(0 \leqq u, v<1)$ for the two genotypes. Then the transmission functions are

$$
f(y)=(1-u) y, \quad g(y)=(1-v)(1-y) .
$$




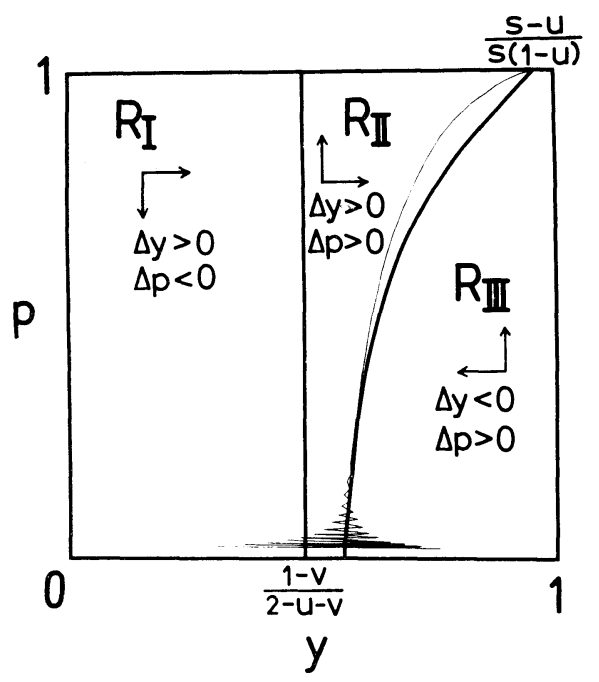

Fig. 1. Error prone imitativeness versus error prone contrariness. Parameters satisfy the inequality $(1-s)(1-u v)-(1-u)^{2}<0$. Figure is drawn for values $u=.04, v=.1, s=.5$. The regions of the variable space are defined as follows. $R_{I}=\{(y, p): 0 \leq y<(1-v) /(2-u$ $-v), 0<p<1\}, R_{I I}=\left\{(y, p):(1-v) /(2-u-v)<y<k^{-1}(p)\right.$, $0<p<1\}, R_{I I I}=\left\{(y, p): k^{-1}(p)<y \leq 1,0<p<1\right\}$, where the rectangular hyperbola $k(y)=[(1-s) y-(1-v)(1-y)$ $(1-s y)] /\{(1-s y)[(2-u-v) y-(1-v)]\}$. In each region a trajectory originating there lies in the indicated quadrant. The boundaries between the regions cause no complications. Note the damped oscillations between $R_{I}$ and $R_{I I I}$, and convergence to the equilibrium $\hat{y}_{2}=(s-u) /[s(1-u)], \hat{p}_{2}=1$. See text for details.

Substituting Eq. (6) into Eqs. (3a) and (3b) and simultaneously setting to 0 , three point equilibria are obtained. At two of these equilibria, $\left(\hat{y}_{1}=0, \hat{p}_{1}=1\right)$ and $\left(\hat{y}_{2}=(s-u) /[s(1-u)], \hat{p}_{2}=1\right)$, genotype $A$ (imitativeness) is fixed. At the third equilibrium, $\left(\hat{y}_{3}, \hat{p}_{3}=0\right)$ where $\hat{y}_{3}$ is the smaller root of the quadratic $m(y) \equiv(1-v) s y^{2}-[2-v(1+s)] y$ $+1-v=0$, genotype $a$ (contrariness) is fixed. Equilibria $\left(\hat{y}_{1}, \hat{p}_{1}\right)$ and $\left(\hat{y}_{3}, \hat{p}_{3}\right)$ exist for all values of the parameters, but $\left(\hat{y}_{2}, \hat{p}_{2}\right)$ exists only for $u<s$.

Local stability analysis using matrix (4) shows that $\left(\hat{y}_{1}, \hat{p}_{1}\right)$ is always unstable. Equilibrium $\left(\hat{y}_{2}, \hat{p}_{2}\right)$ is locally stable if

$$
(1-s)(1-u v)-(1-u)^{2}<0
$$

(which implies $u<s$, i. e. existence), and unstable if

$$
(1-s)(1-u v)-(1-u)^{2}>0 \text {. }
$$

Equilibrium $\left(\hat{y}_{3}, \hat{p}_{3}\right)$ is locally stable if $\hat{y}_{3}<(1-v) /(2-u-v)$ and unstable if the incquality is reversed. By substitution into $m(y)$, it can be 
shown that $(1-v) /(2-u-v)<\hat{y}_{3}<(s-u) /[s(1-u)]$ if $(7 a)$ is satisfied and $(s-u) /[s(1-u)]<\hat{y}_{3}<(1-v) /(2-u-v)$ if $(7 \mathrm{~b})$ is satisfied. Thus, $\left(\hat{y}_{3}, \hat{p}_{3}\right)$ is locally stable if $(7 \mathrm{~b})$ is satisfied and unstable if (7a) is satisfied. In summary, one and only one locally stable equilibrium exists for any set of parameter values (excluding the special borderline case $\left.(1-s)(1-u v)-(1-u)^{2}=0\right)$. Since $\hat{y}_{2}=(s-u) /[s(1-u)]$, inequality (7a) is equivalent to $\hat{y}_{3}<\hat{y}_{2}$, and (7b) to $\hat{y}_{2}<\hat{y}_{3}$. Noting that $\hat{y}_{1}=0$, we can see that the advantageous phenoty pe attains its highest equilibrium frequency at the unique stable equilibrium.

Directions of change in $y$ and $p$ can readily be obtained by applying Eqs. (3a) and (3b), as illustrated in Fig. 1 for the case $(1-s)(1-u v)$ $-(1-u)^{2}<0$ where $\left(\hat{y}_{2}, \hat{p}_{2}\right)$ is locally stable. Oscillations occur in this model, and I have been unable to obtain analytical conditions on global stability. However, numerical iteration suggests that the amplitude of the oscillations are damped (see Fig. 1), and that local stability assures global stability. Although the mean fitness does not increase monotonically, the mean fitness at the stable equilibrium is higher than at other existing equilibria. This can be shown without explicit computation since the curves of the same mean fitness are rectangular hyperbolae with asymptotes $y=(1-v) /(2-u-v)$ and $p=(1-v) /(2--u$ $-v)$.

It is perhaps counterintuitive that contrariness can sometimes prevail over imitativeness. For example, setting $u=v$ in (7b) we have as the condition for $\left(\hat{y}_{s}, \hat{p}_{3}\right)$ to be stable that $u>s /(2-s)$, which holds even when $u<s$. Thus, for small error rates relative to the selection coefficient, imitativeness does better than contrariness, but the advantage can be reversed fairly easily.

I thank Dr. M. Kimura, M. J. A., for suggestions to improve the presentation. Contribution no. 1585 from the National Institute of Genetics, Japan. Supported in part by a Grant-in-Aid for Special Project Research on Biological Aspects of Optimal Strategy and Social Structure from the Japan Ministry of Education, Science and Culture.

\section{References}

1) J. T. Bonner: The Evolution of Culture in Animals. Princeton Univ. Press, Princeton, New Jersey (1980).

2) H. R. Pulliam and C. Dunford: Programmed to Learn. Columbia Univ. Prcss, New York (1980).

3) P. J. Richerson and R. Boyd: J. Social Biol. Struct., 1, 127 (1978).

4) L. L. Cavalli-Sforza and M. W. Feldman: Cultural Transmission and Evolution. Princeton Univ. Press, Princeton, New Jersey (1981).

5) C. J. Lumsden and E. O. Wilson: Genes, Mind, and Culture. Harvard Univ. Press, Cambridge, Mass. (1981).

6) L. L. Cavalli-Sforza and M. W. Feldman: Proc. Natl. Acad. Sci. U.S.A., 80. 4993 (1983).

7) M. W. Feldman and L. L. Cavalli-Sforza: ibid., 81, 1604 (1984). 